\title{
A Relative Dynamics Formulation for Hardware- in-the-loop Simulation of On-orbit Robotic Missions
}

\author{
Marco De Stefano, Hrishik Mishra, Alessandro M. Giordano, Roberto Lampariello and \\ Christian Ott, Senior Member, IEEE
}

\begin{abstract}
In this paper, we propose a novel method for the on-ground simulation of a space robotic mission, while exploiting a robotic hardware-in-the-loop facility. The simulated dynamics replicated by the robots are defined with respect to a nominal motion. This approach enables simulating the motion of large satellites. In particular, we exploit a Lagrangian matching relative to the nominal motion for a satellite (client) and a manipulatorequipped spacecraft (servicer), which are modeled as a rigid body and a multi-body system, respectively. Stability of the proposed method is also analyzed. The effectiveness of the method is demonstrated through experiments on the OOS-Sim facility for the capture of Envisat, a free-tumbling satellite and the largest space debris in Low-Earth-Orbit.
\end{abstract}

Index Terms-Space Robotics and Automation, Dynamics.

\section{INTRODUCTION}

$\mathbf{O}$ $\mathrm{N}$-ORBIT robotic missions are a new class of space missions in which a spacecraft, equipped with a manipulator arm (servicer), is launched into the orbit of a defective satellite (client) to perform a maintenance task or active debris removal. Within the latter context, the manipulator arm is used to approach and grasp the non-cooperative client in order to perform its de-orbit maneuver. A challenging task is the removal of Envisat, an eight-ton Earth-monitoring satellite that is defunct and tumbling freely in space [1]. Different control techniques to dispose Envisat have been proposed in several mission studies such as e.Deorbit [2] and COMRADE (COntrol and Management of Robotics Active DEbris removal) [3], both projects commissioned by the European Space Agency (ESA). Prior to the real space mission, however, the on-board software (OBSW) controller for the servicing maneuver needs to be validated and tested on ground. Therefore, a facility which can replicate the space dynamics conditions on ground is required.

A simulation technology with hardware-in-the-loop (HIL) is recognized as an attractive solution for the on-ground validation of such missions [4]. This technique employs the combination of a dynamic model and a hardware system

Manuscript received: October, 15, 2020; Revised January, 20, 2021; Accepted February, 15, 2021.

This letter was recommended for publication by Editor Jingang Yi upon evaluation of the Associate Editor and Reviewers' comments.

This work has been partially funded by the ESA COMRADE project.

Marco De Stefano, Hrishik Mishra, Alessandro M. Giordano, Roberto Lampariello and Christian Ott are with the Institute of Robotics and Mechatronics, German Aerospace Center (DLR), 82234 Wessling, Germany (email: marco.destefano@dlr.de; hrishik.mishra@dlr.de; alessandro.giordano@dlr.de; roberto.lampariello@dlr.de; christian.ott@dlr.de).

Marco De Stefano and Hrishik Mishra contributed equally to this work.

Digital Object Identifier (DOI): see top of this page.

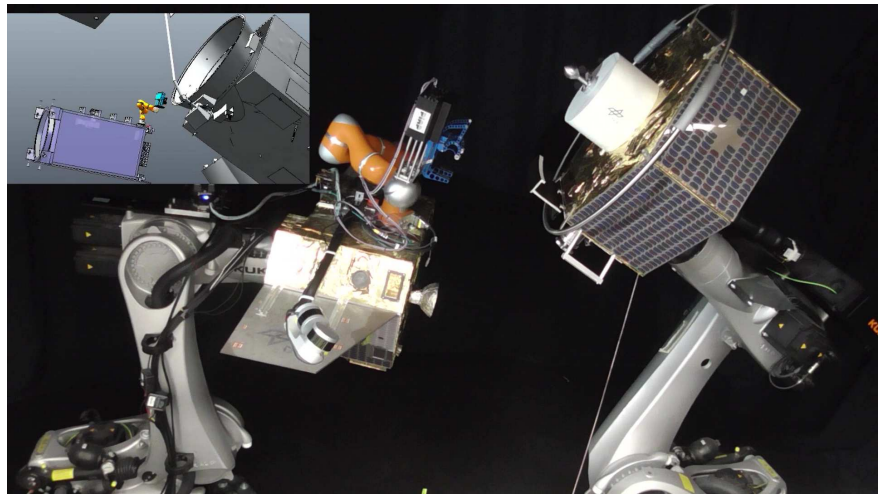

Fig. 1: OOS-Sim facility composed of servicer robot (left) equipped with a light-weight manipulator arm and client robot (right). Top-left: the simulated space scenario for the capture of Envisat.

to reproduce the desired behavior. The sensor measurements available from the HIL facility are exploited by the OBSW for its validation. Usually, robot(s) equipped with mock-ups of a spacecraft and force-torque sensors for measuring the external interaction are exploited within this technique. Several robotics facilities are available for simulating satellite dynamics onground (see [4] for a survey) and an example is the OOSSim facility [5] shown in Fig. 1. Within the OOS-Sim, two industrial robots commanded in admittance-mode replicate the dynamics of the two spacecraft, namely the servicer (left) and the client (right). The servicer is equipped with a light-weight manipulator arm, which performs the capture task.

A typical approach to replicate a desired satellite dynamics with a robotic system is to use an absolute formulation, i.e., the motion of the simulated body with respect to an inertial frame is commanded exactly on a HIL facility with respect to its inertial frame [5], [6], [7], [8]. In contrast, in a relative formulation, the motion in the HIL facility only reflects the relative motion between the two satellites [9], [10], [11]. An inertia shaping approach is proposed in [6] to match the dynamics of the satellite with the mock-up placed on a facility controlled in impedance mode. The key idea is to impose identical acceleration constraints, thereby ensuring identical equations of motion for the satellite and the on-ground robot. This method is called Lagrangian matching [12] or impedance control [13], which will be exploited in this paper.

For replicating the servicer (modeled as multi-body system) on a HIL facility, [14] proposes a partial simulation where joint positions are the input to a fixed-base admittance-controlled robot and the reaction motion of the spacecraft base is only 
numerically simulated. In [15] the reaction motion of the base is emulated by a second robot and the simulated dynamics is based on momentum conservation. The latter is extended by [5] to include the fully actuated dynamics and a torquecontrolled manipulator arm. In [8], a model-based interaction dynamics is considered for the grasping of a client. In [10], [11], the simulation is achieved through a relative motion between a satellite mock-up and a fixed-base manipulator, however, only at kinematic level.

The previous works on absolute representation of the dynamics can have limited applicability when replicating the tumbling velocity of a large satellite (e.g. Envisat) due to workspace constraints. Although a kinematics scaling might be useful to address this problem, however, it will lead to a non-physical simulation because model-based and measured forces will be different. Therefore, a relative formulation of the dynamics is an attractive solution and it defines the scope of this paper.

The contribution of this paper is threefold. Firstly, we address the workspace limitation by simulating the dynamics replicated on the robots with respect to a nominal motion (trajectory). In contrast to prior work on relative dynamics implementations in [10], [11], the proposed method ensures dynamic consistency. Specifically, the fictitious forces of the space analogue scenario, e.g. Coriolis, are experienced on the HIL facility by commanding additional feed-forward terms. To this end, we perform a Lagrangian matching relative to the nominal motion for a client and a servicer satellite simulated on-ground with a HIL robotic facility. The client and the servicer are modeled as a rigid body and a multi-body system, respectively. We provide an impedance and admittance characterization of the proposed relative dynamics formulation for the client and servicer. Secondly, through stability analysis, we prove the boundedness of the velocity for the HIL facility. Finally, we verify the proposed method with a state-of-theart dynamics simulator, namely GNCDE [16] and we prove the effectiveness of the method through experiments on the OOS-Sim facility for the capture of the Envisat satellite.

The paper is structured as follows: Sec. II introduces the problem statement, the proposed method with the relative dynamics formulation is presented in Sec. III and the stability analysis in Sec. IV. The experimental validation is presented in Sec. V and Sec. VI concludes the paper.

\section{Dynamics Simulation With HIL FaCility}

Industrial robots are a key technology for a robotic HIL facility to simulate a desired dynamics and a suitable control strategy is the admittance control [5]. Specifically, a reduced admittance architecture is shown in Fig. 2. The industrial robot is equipped with a force-torque sensor to measure the interaction wrenches with the environment, $\boldsymbol{F}$, which is the input to a desired dynamic model (Des. Dyn.). Its output is an acceleration that after double integration $\left(\iint\right)$ results in a desired pose, $\boldsymbol{g}$, which is then commanded to the industrial robot via inverse kinematics control.

We indicate in this paper, a pose as $\boldsymbol{g}_{(\bullet)}=\left(\boldsymbol{R}_{(\bullet)}, \boldsymbol{p}_{(\bullet)}\right) \in$ $S E(3)$, where $\boldsymbol{R}$ and $\boldsymbol{p}$ are the rotation matrix and the position,

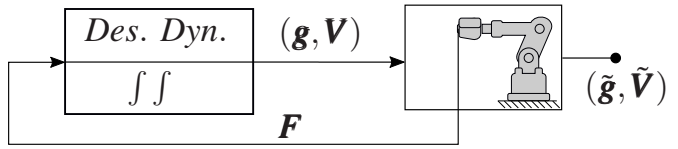

Fig. 2: Admittance scheme with the desired dynamic model.

respectively (see (1)) and the differential kinematics of a pose is given as $\dot{\boldsymbol{g}}_{(\bullet)}=\boldsymbol{g}_{(\bullet)} \boldsymbol{V}_{(\bullet \bullet}^{\wedge}$, where $\boldsymbol{V}_{(\bullet)} \in \mathbb{R}^{6} \cong s e(3)$ is the body velocity $\boldsymbol{V}_{(\bullet)}=\left[\begin{array}{ll}\boldsymbol{v}_{(\bullet)}^{T} & \boldsymbol{\omega}_{(\bullet)}^{T}\end{array}\right]^{T}$ consisting of linear $(\boldsymbol{v})$ and angular $(\boldsymbol{\omega})$ velocities and the operator $(\bullet)^{\wedge}: \operatorname{se}(3) \rightarrow \mathbb{R}^{6}$ is given in (1)

$$
\boldsymbol{g}_{(\bullet)}=\left[\begin{array}{cc}
\boldsymbol{R}_{(\bullet)} & \boldsymbol{p}_{(\bullet)} \\
0 & 1
\end{array}\right], \boldsymbol{V}_{(\bullet)}^{\wedge}=\left[\begin{array}{cc}
\boldsymbol{\omega}_{(\bullet) \times} & \boldsymbol{v}_{(\bullet)} \\
0 & 0
\end{array}\right],
$$

where $(\bullet)_{\times}$is the skew-symmetric matrix of the vector argument $(\bullet)$, see [17] for details. Furthermore, the following assumption is considered.

Assumption 1: The industrial robot provides a perfect tracking, i.e. the measurement output is the same as the commands, $(\tilde{\boldsymbol{g}}, \tilde{\boldsymbol{V}}) \sim(\boldsymbol{g}, \boldsymbol{V})$ in Fig. 2.

This is common for industrial robots, in which the gains of the low-level control are high enough to achieve high position accuracy.

Problem Statement: The desired dynamics (Des. Dyn) in Fig. 2 represents the behavior to be simulated and it is usually expressed as the dynamics of a rigid body with desired mass and inertia parameters in an absolute form [6], [18]. This means that the motion (represented by the pose $\boldsymbol{g}$ in Fig. 2) of the simulated body is commanded exactly to the industrial robot with respect to an inertial frame.

To faithfully simulate the grasping task for a servicing mission, the kinematic structure of the satellites must also be considered, e.g. the location of the grasping point with respect to the satellite center of mass (Com). For large satellite structures, the Com can be located at a large distance from the defined grasping point. This factor can limit the range of simulations on-ground, especially when fast angular velocities are considered. An example is the Envisat satellite, a large space debris tumbling in orbit, whose Com is located at a distance of $2.9 \mathrm{~m}$ from the grasping point [1]. It has been estimated that the current angular velocity of Envisat is $2.5 \mathrm{deg} / \mathrm{s}$ about its major axis of inertia [3]. Considering the geometry of Envisat and the given angular velocity, the industrial robot during the HIL simulation will be subjected to move along an arc-length, as shown in Fig. 3.

The absolute formulation of the dynamics reproduced with a HIL facility leads to operational limits such as workspace limitation or limited time duration for the simulation. This factor might not allow the verification of the complete capture maneuver. For example, only 14 seconds of experiment were allowed by the client robot at the OOS-Sim facility before encountering workspace limits of the robots. For usual grasping maneuver, a longer time is required to approach the client satellite (see e.g. [3]) and a solution is required for validating the complete control algorithm on ground, prior to the launch. 


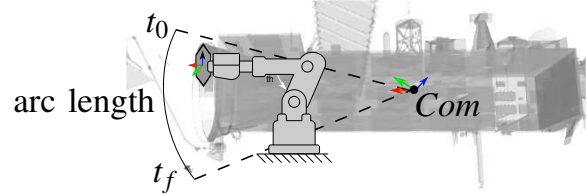

Fig. 3: HIL simulation of Envisat: the absolute dynamics representation limits the replication of motion of large satellite structures with large angular velocity on a robotic facility.

\section{PROPOSED METHOD}

The proposed method is based on a relative dynamics formulation with respect to a nominal trajectory (later defined), which is parameterized as a pose $\boldsymbol{g}_{\boldsymbol{n}}$, velocity $\boldsymbol{V}_{\boldsymbol{n}}=\left[\boldsymbol{v}_{\boldsymbol{n}}, \boldsymbol{\omega}_{\boldsymbol{n}}\right]$ and acceleration $\dot{\boldsymbol{V}}_{n}$. Firstly, the case of a rigid body, i.e. the client, is tackled and later extended for the multi-body system, i.e. the servicer. For completeness, the treatment will be provided for a robot controlled in two modalities, i.e. impedance causality (velocity input/force output) and admittance causality (force input/velocity output). The main focus will be on the admittance-controlled robot, since it represents a common control scheme for simulating a desired dynamics with robots [5], [9].

\section{A. Rigid body dynamics for the client in HIL}

The satellite dynamics to be simulated by the client robot is that of a rigid body, expressed as,

$$
M_{t} \dot{V}_{t}+C_{t}\left(V_{t}\right) V_{t}=F_{t}
$$

where $\boldsymbol{M}_{\boldsymbol{t}} \in \mathbb{R}^{6 \times 6}$ is the inertia matrix, $\boldsymbol{C}_{\boldsymbol{t}} \in \mathbb{R}^{6 \times 6}$ is the nonlinear Coriolis/centrifugal matrix, $\dot{\boldsymbol{V}}_{\boldsymbol{t}} \in \mathbb{R}^{6}$ and $\boldsymbol{V}_{\boldsymbol{t}} \in \mathbb{R}^{6}$ are the acceleration and velocity, respectively. $\boldsymbol{F}_{\boldsymbol{t}} \in \mathbb{R}^{6}$ is the body external wrench vector around the Com.

The dynamic in (2) needs to be matched with the robot dynamics, whose Cartesian dynamics is:

$$
\boldsymbol{M}_{c} \dot{\boldsymbol{V}}_{c}+\boldsymbol{C}_{\boldsymbol{c}}\left(\boldsymbol{V}_{\boldsymbol{c}}\right) \boldsymbol{V}_{\boldsymbol{c}}=\tilde{\boldsymbol{F}}_{\boldsymbol{c}}+\boldsymbol{F}_{\boldsymbol{c}},
$$

where the subscript $\boldsymbol{c}$ for the matrices and vectors in (3) refers now to the robot (client). $\boldsymbol{F}_{c}$ is the input control wrench and $\tilde{F_{c}}$ is the external wrench at the Com. The external wrench is measured by a force-torque sensor located at the end-effector of the industrial robot and transformed to the Com frame using a constant transformation, which does not affect the dynamics. We assume that the dynamics in (3) is already compensated with the gravitational forces.

In a classical Lagrangian matching the relative acceleration (between $\dot{V}_{c}$ and $\dot{V}_{t}$ ) is set to zero [6], however this results in the replication of absolute dynamics on the hardware, which is the key problem. The main idea is to command to the robot a motion relative to a nominal trajectory. This is schematically represented in Fig. 4, where the line in red denotes the simulated motion of the spacecraft in (2) and the line in green is a nominal trajectory. For example, the unforced motion of the client could be considered as,

$$
M_{t} \dot{V}_{n}+C_{t}\left(V_{n}\right) V_{n}=\mathbf{0},
$$

where the initial condition $\boldsymbol{V}_{\boldsymbol{n}}(\mathbf{0})$ is the same as the mission requirement, $V_{t}(\mathbf{0})$. Therefore, the commanded motion to the

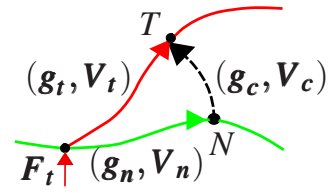

Fig. 4: Nominal trajectory $\left(g_{n}, V_{n}\right)$, forced trajectory $\left(g_{t}, V_{t}\right)$, relative trajectory commanded to the robot, $\left(g_{c}, V_{c}\right)$.

robot will be $\left(\boldsymbol{g}_{c}, \boldsymbol{V}_{\boldsymbol{c}}\right)$, as shown in Fig. 4 with a dashed line. From the pose relationship in Fig. $4, \boldsymbol{g}_{\boldsymbol{t}}=\boldsymbol{g}_{\boldsymbol{n}} \boldsymbol{g}_{\boldsymbol{c}}$ and taking the time derivative pre-multiplied with $\boldsymbol{g}_{t}^{-1}$, we get,

$$
\boldsymbol{g}_{t}^{-1}\left(\dot{\boldsymbol{g}}_{\boldsymbol{n}} \boldsymbol{g}_{\boldsymbol{c}}+\boldsymbol{g}_{\boldsymbol{n}} \dot{\boldsymbol{g}}_{\boldsymbol{c}}\right)=\boldsymbol{V}_{\boldsymbol{t}}^{\wedge} \Longrightarrow \boldsymbol{V}_{\boldsymbol{c}}^{\wedge}+\boldsymbol{g}_{c}^{-1} \boldsymbol{V}_{\boldsymbol{n}}^{\wedge} \boldsymbol{g}_{\boldsymbol{c}}=\boldsymbol{V}_{\boldsymbol{t}}^{\wedge}
$$

where $\dot{\boldsymbol{g}}_{\boldsymbol{n}}=\boldsymbol{g}_{n} \boldsymbol{V}_{\boldsymbol{n}}^{\wedge}$ and $\dot{\boldsymbol{g}}_{\boldsymbol{c}}=\boldsymbol{g}_{c} \boldsymbol{V}_{\boldsymbol{c}}^{\wedge}$ have been substituted. Considering that $\boldsymbol{g}_{\boldsymbol{c}}^{-1} \boldsymbol{V}_{\boldsymbol{n}}^{\wedge} \boldsymbol{g}_{\boldsymbol{c}}=\left(\boldsymbol{A d}_{\boldsymbol{g}_{c}^{-1}} \boldsymbol{V}_{\boldsymbol{n}}\right)^{\wedge}$, the following relation holds,

$$
\boldsymbol{V}_{c}+\underbrace{\boldsymbol{A d _ { g _ { c } ^ { - 1 } } V _ { n }}}_{\Delta \boldsymbol{V}_{c}}=V_{\boldsymbol{t}},
$$

where the term $\boldsymbol{A d}_{\boldsymbol{g}_{\boldsymbol{c}}^{-1}}$ transforms ${ }^{1}$ the body-velocity $\boldsymbol{V}_{\boldsymbol{n}}$ from $\boldsymbol{g}_{\boldsymbol{n}}$ to $\boldsymbol{g}_{\boldsymbol{t}}$. Therefore, we propose the novel Lagrangian matching condition by taking the time derivative of (6), as

$$
\left(\dot{\boldsymbol{V}}_{\boldsymbol{t}}-\dot{\boldsymbol{V}}_{\boldsymbol{c}}\right)=\Delta \dot{\boldsymbol{V}}_{\boldsymbol{c}}
$$

where $\Delta \dot{V}_{c}$ is the acceleration between the simulated trajectory and the HIL robot.

1) Impedance Causality: In this modality, the scope is to find the input, $\boldsymbol{F}_{\boldsymbol{c}}$, to the impedance-controlled robot whose dynamics are given in (3). Therefore from (7), it follows that $\boldsymbol{M}_{\boldsymbol{c}}\left(\dot{\boldsymbol{V}}_{\boldsymbol{t}}-\dot{\boldsymbol{V}}_{\boldsymbol{c}}\right)=\boldsymbol{M}_{\boldsymbol{c}} \Delta \dot{\boldsymbol{V}}_{\boldsymbol{c}}$ and by substituting $\dot{\boldsymbol{V}}_{\boldsymbol{t}}$ from (2) and $\dot{V}_{c}$ from (3), we get:

$$
\begin{aligned}
\boldsymbol{F}_{\boldsymbol{c}}-\boldsymbol{M}_{c} \boldsymbol{M}_{t}^{-1} \boldsymbol{F}_{\boldsymbol{t}} & =-\tilde{\boldsymbol{F}}_{\boldsymbol{c}}-\boldsymbol{M}_{\boldsymbol{c}} \Delta \dot{\boldsymbol{V}}_{\boldsymbol{c}} \\
& -\boldsymbol{M}_{\boldsymbol{c}} \boldsymbol{M}_{t}^{-1} \boldsymbol{C}_{\boldsymbol{t}}\left(\boldsymbol{V}_{t}\right) \boldsymbol{V}_{\boldsymbol{t}}+\boldsymbol{C}_{\boldsymbol{c}}\left(\boldsymbol{V}_{\boldsymbol{c}}\right) \boldsymbol{V}_{\boldsymbol{c}} .
\end{aligned}
$$

In (8), the interaction force $\boldsymbol{F}_{\boldsymbol{t}}=\tilde{\boldsymbol{F}_{\boldsymbol{c}}}$ and it follows that,

$$
\begin{aligned}
\boldsymbol{F}_{\boldsymbol{c}} & =\left(\boldsymbol{M}_{\boldsymbol{c}} \boldsymbol{M}_{t}^{-1}-\boldsymbol{I}\right) \tilde{\boldsymbol{F}}_{\boldsymbol{c}}-\boldsymbol{M}_{\boldsymbol{c}} \Delta \dot{\boldsymbol{V}}_{\boldsymbol{c}} \\
& -\boldsymbol{M}_{\boldsymbol{c}} \boldsymbol{M}_{\boldsymbol{t}}^{-1} \boldsymbol{C}_{\boldsymbol{t}}\left(\boldsymbol{V}_{\boldsymbol{t}}\right) \boldsymbol{V}_{\boldsymbol{t}}+\boldsymbol{C}_{\boldsymbol{c}}\left(\boldsymbol{V}_{\boldsymbol{c}}\right) \boldsymbol{V}_{\boldsymbol{c}} .
\end{aligned}
$$

Eq. (9) is the input to the impedance-controlled robot in (3), which allows to reproduce the relative dynamics as in (7).

2) Admittance Causality: For an admittance-controlled robot, the inertia is a parameter and it can be set equal to the desired one to be simulated, i.e. $\boldsymbol{M}_{\boldsymbol{c}}=\boldsymbol{M}_{\boldsymbol{t}}$. Therefore (9) simplifies as:

$$
\boldsymbol{F}_{\boldsymbol{c}}=-\boldsymbol{M}_{t} \Delta \dot{\boldsymbol{V}}_{\boldsymbol{c}}-\boldsymbol{C}_{\boldsymbol{t}}\left(\boldsymbol{V}_{\boldsymbol{t}}\right) \boldsymbol{V}_{\boldsymbol{t}}+\boldsymbol{C}_{\boldsymbol{c}}\left(\boldsymbol{V}_{\boldsymbol{c}}\right) \boldsymbol{V}_{\boldsymbol{c}} .
$$

Then, by substituting (10) in (3), the following admittance dynamics equation can be found:

$$
\boldsymbol{M}_{t} \dot{\boldsymbol{V}}_{\boldsymbol{c}}+\boldsymbol{C}_{\boldsymbol{t}}\left(\boldsymbol{V}_{t}\right) \boldsymbol{V}_{\boldsymbol{t}}=\tilde{\boldsymbol{F}}_{\boldsymbol{c}}-\boldsymbol{M}_{\boldsymbol{t}} \Delta \dot{\boldsymbol{V}}_{\boldsymbol{c}} .
$$

Eq. (11) represents the equation to be integrated (with initial velocity $\boldsymbol{V}_{\boldsymbol{c}}(0)=\mathbf{0}$ ) in order to command a pose, $\boldsymbol{g}_{\boldsymbol{c}}$ to the industrial robot, which simulates the desired relative dynamics. Note that (11) includes the Coriolis wrench from the simulated

$$
{ }^{1} \text { For a generic pose } \boldsymbol{g}_{(\bullet)} \text {, it is defined as, } \mathbf{A d}_{g_{(\bullet)}}=\left[\begin{array}{cc}
\boldsymbol{R}_{(\bullet)} & \boldsymbol{p}_{(\bullet \bullet} \times \boldsymbol{R}_{(\bullet)} \\
0 & \boldsymbol{R}_{(\bullet)}
\end{array}\right] \text {. }
$$




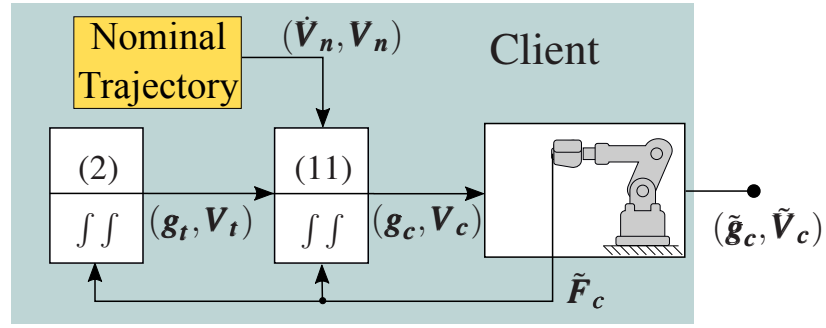

Fig. 5: Admittance scheme for simulating a client satellite on the HIL with the proposed method, where $\boldsymbol{A} \boldsymbol{d}_{\boldsymbol{g}_{c}^{-1}} \boldsymbol{V}_{\boldsymbol{n}}=\Delta \boldsymbol{V}_{\boldsymbol{c}}$ is used in (11).

scenario in orbit, i.e. $\boldsymbol{C}_{\boldsymbol{t}} \boldsymbol{V}_{\boldsymbol{t}}$, to the simulated scenario onground considering also the measured force on the hardware, $\tilde{\boldsymbol{F}}_{\boldsymbol{c}}$ and the inertial force $\boldsymbol{M}_{\boldsymbol{t}} \Delta \dot{\boldsymbol{V}}_{\boldsymbol{c}}$. Fig. 5 shows the proposed schematic to perform the dynamics matching for simulating the relative dynamics on an admittance-controlled robot.

\section{B. Multi-body dynamics for the servicer in HIL}

Let us introduce the simulated dynamics of a gravity-free manipulator arm with $n$-joints mounted on a servicer satellite (base) as follows,

$$
\begin{aligned}
{\left[\begin{array}{cc}
M_{b} & M_{b q} \\
M_{b q}^{T} & M_{q}
\end{array}\right]\left[\begin{array}{c}
\dot{V}_{b} \\
\ddot{q}
\end{array}\right]+} & {\left[\begin{array}{cc}
C_{b} & C_{b q} \\
C_{q b} & C_{q}
\end{array}\right]\left[\begin{array}{c}
V_{b} \\
\dot{q}
\end{array}\right]=} \\
& {\left[\begin{array}{c}
F_{b} \\
\tau
\end{array}\right]+\left[\begin{array}{c}
J_{b}^{T} \\
J_{m}^{T}
\end{array}\right] F_{e} }
\end{aligned}
$$

where $\boldsymbol{M}_{\boldsymbol{b}} \in \mathbb{R}^{6 \times 6}, \boldsymbol{M}_{\boldsymbol{q}} \in \mathbb{R}^{n \times n}, \boldsymbol{M}_{\boldsymbol{b q}} \in \mathbb{R}^{6 \times n}$ are the inertia matrices of the whole servicer spacecraft, manipulator and the coupling between the base and the manipulator, respectively. $\boldsymbol{C}_{\boldsymbol{b}} \in \mathbb{R}^{6 \times 6}, \boldsymbol{C}_{\boldsymbol{q}} \in \mathbb{R}^{n \times n}$ and $\boldsymbol{C}_{\boldsymbol{q} \boldsymbol{m}} \in \mathbb{R}^{6 \times n}$ are the nonlinear Coriolis/centrifugal matrix of the base, manipulator and coupling between base-manipulator, respectively. The vectors $\dot{\boldsymbol{V}}_{\boldsymbol{b}} \in \mathbb{R}^{6}$ and $\ddot{\boldsymbol{q}} \in \mathbb{R}^{n}$ are the acceleration of the base (linear and angular) and the acceleration of the robot joints respectively and, $V_{\boldsymbol{b}} \in \mathbb{R}^{6}$ and $\dot{\boldsymbol{q}} \in \mathbb{R}^{n}$ are the respective velocity vectors. $\boldsymbol{F}_{\boldsymbol{b}} \in \mathbb{R}^{6}$ is the force-torque wrench generated by the satellite actuation and acting on the Com and $\tau \in \mathbb{R}^{n}$ is the input torque vector to the manipulator. $\boldsymbol{J}_{\boldsymbol{b}} \in \mathbb{R}^{6 \times 6}$ and $\boldsymbol{J}_{\boldsymbol{m}} \in \mathbb{R}^{6 \times n}$ are the Jacobian matrices of the base and the manipulator, $\boldsymbol{F}_{\boldsymbol{e}} \in \mathbb{R}^{6}$ is the external wrench acting at the end-effector.

Usually, the first line in (12) is exploited to simulate the dynamics of the base, commanding the resulting $\boldsymbol{V}_{\boldsymbol{b}}$ to the industrial robot [5] and we report it in explicit form as,

$$
M_{b} \dot{V}_{b}+M_{b q} \ddot{q}+C_{b} V_{b}+C_{b q} \dot{q}=F_{b}+J_{b}^{T} F_{e} .
$$

To match the dynamics, we need to introduce the servicer robot equation, whose matrices and vectors will be indicated with the subscript $\boldsymbol{s}$ in the upcoming equations. Therefore,

$$
M_{s} \dot{V}_{s}+M_{s q} \ddot{q}+C_{s} V_{s}+C_{s q} \dot{q}=F_{s}+J_{s}^{T} \tilde{F}_{e}
$$

where $\boldsymbol{F}_{\boldsymbol{s}}$ is the input actuation control force and $\tilde{\boldsymbol{F}}_{\boldsymbol{e}}$ is the external wrench measured by a force-torque sensor. We assume that the dynamics in (14) is already compensated with the gravitational forces.
Assumption 2: The servicer manipulator arm on ground and the space manipulator arm have the same kinematics, inertial parameters and initial values of the position and velocity in the joints.

Assumption 2 implies that the Jacobian matrix $\boldsymbol{J}_{\boldsymbol{b}}$ in (13) is identical to $\boldsymbol{J}_{\boldsymbol{s}}$ in (14). Additionally, the coupling inertia will be the same, i.e. $\boldsymbol{M}_{b q}=\boldsymbol{M}_{s q}$ because they depend on the joint states. Following the treatment introduced in the Sec. III-A with the nominal trajectory, the velocity relationship is imposed for the servicer and the proposed Lagrangian matching condition results as,

$$
V_{s}+\underbrace{A d_{g_{s}^{-1}} V_{n}}_{\Delta V_{s}}=V_{b} \Rightarrow M_{b}\left(\dot{V}_{b}-\dot{V}_{s}\right)=M_{b} \Delta \dot{V}_{s}
$$

where $\Delta \boldsymbol{V}_{s}=\boldsymbol{A} \boldsymbol{d}_{g_{s}^{-1}} \boldsymbol{V}_{\boldsymbol{n}}$ and it represents the velocity between the simulated dynamics $\boldsymbol{V}_{\boldsymbol{b}}$ and the hardware $\boldsymbol{V}_{\boldsymbol{s}}$.

1) Impedance Causality: In this modality, we need to find $\boldsymbol{F}_{\boldsymbol{s}}$ as input to the robot in (14). Then, by substituting $\dot{\boldsymbol{V}}_{\boldsymbol{b}}$ from (13) and $\dot{V}_{s}$ from (14) in (15) while considering Assumption 2, the actuation force $\boldsymbol{F}_{\boldsymbol{s}}$ results in,

$$
\begin{aligned}
\boldsymbol{F}_{s} & -\boldsymbol{M}_{\boldsymbol{s}} \boldsymbol{M}_{\boldsymbol{b}}^{-1} \boldsymbol{J}_{b}^{T} \boldsymbol{F}_{\boldsymbol{e}}=\boldsymbol{C}_{\boldsymbol{s}} \boldsymbol{V}_{\boldsymbol{s}}+\left(\boldsymbol{C}_{s q}-\boldsymbol{M}_{\boldsymbol{s}} \boldsymbol{M}_{b}^{-1} \boldsymbol{C}_{\boldsymbol{b q}}\right) \dot{\boldsymbol{q}} \\
& +\boldsymbol{M}_{\boldsymbol{s}} \boldsymbol{M}_{\boldsymbol{b}}^{-1}\left(\boldsymbol{F}_{\boldsymbol{b}}+\boldsymbol{J}_{\boldsymbol{b}}^{T} \tilde{\boldsymbol{F}}_{\boldsymbol{e}}-\boldsymbol{C}_{\boldsymbol{b}} \boldsymbol{V}_{\boldsymbol{b}}-\boldsymbol{M}_{\boldsymbol{b}} \Delta \dot{\boldsymbol{V}}_{\boldsymbol{s}}\right) .
\end{aligned}
$$

In (16), the interaction wrench is chosen as, $\boldsymbol{F}_{\boldsymbol{e}}=\tilde{\boldsymbol{F}}_{\boldsymbol{e}}$ and the input to the dynamics of the robot in (14), $\boldsymbol{F}_{\boldsymbol{s}}$, is derived.

2) Admittance Causality: In this mode, the inertia of the servicer robot $\boldsymbol{M}_{\boldsymbol{s}}$ is equal to the simulated mass $\boldsymbol{M}_{\boldsymbol{b}}$. Therefore, (16) simplifies as:

$$
\boldsymbol{F}_{\boldsymbol{s}}=\boldsymbol{C}_{\boldsymbol{s}} \boldsymbol{V}_{\boldsymbol{s}}-\boldsymbol{C}_{b} \boldsymbol{V}_{\boldsymbol{b}}+\left(\boldsymbol{C}_{\boldsymbol{s} q}-\boldsymbol{C}_{b q}\right) \dot{\boldsymbol{q}}+\boldsymbol{F}_{\boldsymbol{b}}-\boldsymbol{M}_{b} \Delta \dot{\boldsymbol{V}}_{\boldsymbol{s}},
$$

where $\boldsymbol{F}_{\boldsymbol{e}}=\tilde{\boldsymbol{F}}_{\boldsymbol{e}}$ has been considered. By substituting (17) in (14) and considering Assumption 2, we obtain the admittancetype dynamics to simulate on the servicer robot, as:

$$
M_{b} \dot{V}_{s}+M_{b q} \ddot{q}+C_{b} V_{b}+C_{b q} \dot{q}=J_{b}^{T} \tilde{F}_{e}+F_{b}-M_{b} \Delta \dot{V}_{s}
$$

Eq. (18) is the equation to be integrated in $\dot{\boldsymbol{V}}_{\boldsymbol{s}}$ to obtain a pose to be commanded to the industrial robot, $g_{s}$. Note that in (18), the Coriolis forces from the simulated scenario are mapped into the dynamics that the robot will reproduce. Furthermore, $\boldsymbol{F}_{\boldsymbol{b}}$ will be the actuation force input provided by the OBSW controller. Further note that in the proposed Lagrangian matching, the inertial acceleration of the nominal trajectory appears, as it can be seen in the last term of (18) and (11), respectively.

\section{Servicer manipulator arm}

For a faithful on-ground simulation, the fictitious torques for the manipulator arm, e.g. Coriolis, need to be mapped from the space analogue scenario and commanded to the manipulator on ground, as feed-forward terms. To this end, let us introduce the dynamics of the manipulator in orbit, which is dictated by the second line of (12), as follows:

$$
M_{b q}^{T} \dot{V}_{b}+M_{q} \ddot{q}+C_{q b}\left(\dot{q}, V_{b}\right) V_{b}+C_{q}\left(\dot{q}, V_{b}\right) \dot{q}=\tau+J_{m}^{T} F_{e},
$$

where we made explicit the dependency in the Coriolis terms. 
Considering Assumption 2, the real manipulator arm dynamics can be expressed as:

$M_{b q}^{T} \dot{V}_{s}+M_{q} \ddot{q}+C_{q s}\left(\dot{q}, V_{s}\right) V_{s}+C_{q}\left(\dot{q}, V_{s}\right) \dot{q}=\tau_{q}+J_{m}^{T} \tilde{F}_{e}+G$

where $\tau_{q} \in \mathbb{R}^{n}$ is the actuation joint torque, $\tilde{\boldsymbol{F}}_{\boldsymbol{e}}$ is the external force vector measured at the end-effector and $\boldsymbol{G} \in \mathbb{R}^{n}$ is the gravity vector. Note that for the real manipulator, the Coriolis terms in (20) are a function of the HIL servicer velocity, $\boldsymbol{V}_{\boldsymbol{s}}$.

From (19) and (20), it is possible to obtain the required control action. In particular, we perform a perfect Lagrangian matching, i.e. the relative acceleration between the manipulator arm in space and the one on-ground are set to zero, as

$$
\left(\boldsymbol{M}_{q} \ddot{\boldsymbol{q}}\right)_{f r o m(19)}-\left(\boldsymbol{M}_{q} \ddot{\boldsymbol{q}}\right)_{f r o m(20)}=\mathbf{0}
$$

Substituting the corresponding dynamics in (21) and noting that $\boldsymbol{F}_{\boldsymbol{e}}=\widetilde{\boldsymbol{F}}_{\boldsymbol{e}}$, we obtain the impedance control law [13], as:

$$
\begin{aligned}
\tau_{q}= & \tau-G+\underbrace{\boldsymbol{M}_{\boldsymbol{b}}^{\boldsymbol{T}}\left(\dot{\boldsymbol{V}}_{\boldsymbol{s}}-\dot{\boldsymbol{V}}_{\boldsymbol{b}}\right)}_{\tau_{f f_{1}}}+\underbrace{}_{\tau_{f f_{2}}\left(\boldsymbol{C}_{\boldsymbol{q}}\left(\dot{\boldsymbol{q}}, \boldsymbol{V}_{\boldsymbol{s}}\right)-C_{q}\left(\dot{\boldsymbol{q}}, \boldsymbol{V}_{\boldsymbol{b}}\right)\right) \dot{\boldsymbol{q}}} \\
& +\underbrace{C_{q \boldsymbol{s}}\left(\dot{\boldsymbol{q}}, \boldsymbol{V}_{\boldsymbol{s}}\right) \boldsymbol{V}_{\boldsymbol{s}}-\boldsymbol{C}_{\boldsymbol{q} b}\left(\dot{\boldsymbol{q}}, \boldsymbol{V}_{\boldsymbol{b}}\right) \boldsymbol{V}_{\boldsymbol{b}}}_{\tau_{f f_{3}}} .
\end{aligned}
$$

Eq. (22) is the torque vector commanded to the manipulator arm on-ground compensated with the gravity $\boldsymbol{G}$, while additionally experiencing the same torques as in orbit. In particular, the vector $\tau$ is the input torques of the controller provided by the OBSW. The feed-forward terms, $\tau_{f f_{1}}$ and $\tau_{f f_{3}}$, are acceleration and Coriolis terms between the servicerbase in hardware and the simulated one, respectively, and $\tau_{f f_{2}}$ provides the $\dot{\boldsymbol{q}}$-dependent Coriolis term to match the dynamics of the hardware and the space manipulator arm.

The new schematic for simulating a servicer satellite (modeled as multi-body dynamics) on an admittance-controlled robot equipped with a torque-controlled manipulator arm, is shown in Fig. 6. Note that the measured data from the industrial robot, $\left(\tilde{\boldsymbol{g}}_{\boldsymbol{s}}, \tilde{\boldsymbol{V}}_{\boldsymbol{s}}\right)$, and the manipulator arm, $(\boldsymbol{q}, \dot{\boldsymbol{q}})$, are used to compute equation (13), (18) and (22).

Furthermore, the nodes on $\boldsymbol{F}_{\boldsymbol{b}}$ and $\boldsymbol{\tau}$ in Fig. 6 are the input from the OBSW controller to be validated on the ground.

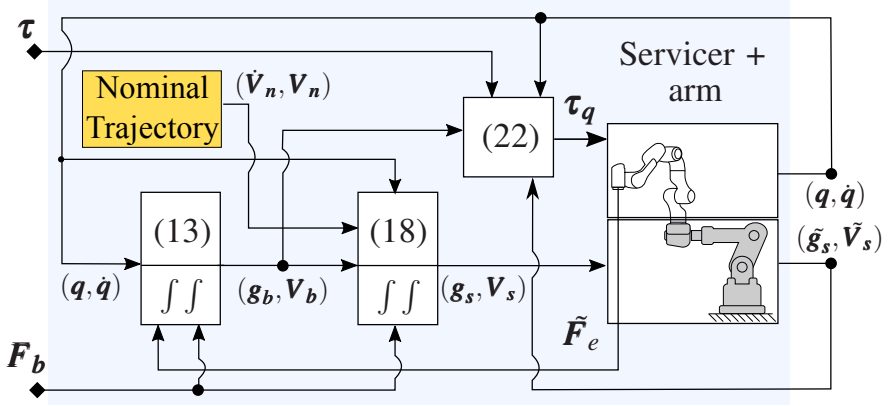

Fig. 6: Admittance scheme for simulating a manipulator-equipped servicer satellite on a HIL facility with the proposed method. Here $\boldsymbol{A} \boldsymbol{d}_{\boldsymbol{g}_{\boldsymbol{s}}^{-1}} \boldsymbol{V}_{\boldsymbol{n}}=\Delta \boldsymbol{V}_{\boldsymbol{s}}$ is used as input in (18).

\section{Final HIL architecture for on-orbit robotic missions}

For a space robotic mission, the servicer and client satellite are in close proximity, in such a way that the manipulator arm interacts with the client. In this case, the interaction occurs between the gripper mounted on the manipulator arm and the grappling structure of the client.

Assumption 3: During interaction, the client experiences a wrench $\boldsymbol{F}_{\boldsymbol{t}}$ and its reaction wrench, namely $\boldsymbol{F}_{\boldsymbol{e}}$ is transmitted to the servicer manipulator $\operatorname{arm}^{2}$ such that $\boldsymbol{F}_{\boldsymbol{e}}=-\boldsymbol{F}_{\boldsymbol{t}}$, i.e. $\boldsymbol{F}_{\boldsymbol{e}}$ and $\boldsymbol{F}_{\boldsymbol{t}}$ are internal wrenches.

Assumption 3 implies that on the HIL facility, $\tilde{\boldsymbol{F}}_{\boldsymbol{e}}=-\tilde{\boldsymbol{F}}_{\boldsymbol{c}}$ and it holds true for the whole grasping phase, which includes the initial contacts of the gripper with the client structure, the closing of the gripper and the post grasping dynamics. The proposed method for the client (shown in Fig. 5) and for the servicer+arm (in Fig. 6), is applicable for simulating onground such a robotic mission. In particular, Fig. 7 summarizes the final architecture and it shows all the proposed elements within the dashed-line, where the input wrenches to the servicer+arm block considers Assumption 3.

The Relative Mapping block in the proposed architecture simply transforms the measurements from the facility (indicated with $(\tilde{\bullet})$ ) to the absolute state measurements of the client and servicer in orbit. These states are required as an input to the Sensor performance block, which consists of relative and absolute sensors (e.g. star-tracker). Usually, these sensors are defined by the space mission specification. The relative mapping will therefore exploit the nominal motion to compute the pose and velocity as follows,

$$
\begin{array}{ll}
\tilde{\boldsymbol{g}}_{t}=\boldsymbol{g}_{n} \tilde{\boldsymbol{g}}_{c} & \tilde{V}_{t}=\tilde{V}_{c}+\Delta V_{c} \\
\tilde{\boldsymbol{g}}_{b}=\boldsymbol{g}_{n} \tilde{\boldsymbol{g}}_{s} & \tilde{V}_{b}=\tilde{V}_{s}+\Delta V_{s}
\end{array}
$$

Note that the initial condition in (2) can be chosen according to the desired space scenario (e.g. for Envisat can be set as

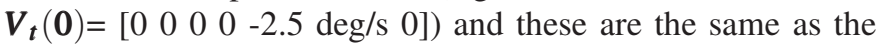
one of the nominal motion in (4), i.e. $V_{t}(\mathbf{0})=V_{n}(\mathbf{0})$. For the commanded data to the client robot in (11), $V_{c}(\mathbf{0})=0$ and for the servicer in (18) the initial condition can be set with respect to the client simulated motion such as, $\boldsymbol{V}_{\boldsymbol{b}}(\mathbf{0})=\boldsymbol{A} \boldsymbol{d}_{\boldsymbol{g}_{b t}} \boldsymbol{V}_{t}(\mathbf{0})$. Then, $\boldsymbol{V}_{\boldsymbol{s}}(\mathbf{0})=\boldsymbol{V}_{\boldsymbol{b}}(\mathbf{0})-\boldsymbol{A} \boldsymbol{d}_{g_{s}^{-1}} \boldsymbol{V}_{\boldsymbol{t}}(\mathbf{0})$.

The OBSW controller can be then validated under realistic dynamics motions to accomplish the on-orbit robotic mission task. A requirement for the OBSW controller in closed-loop with (12) is to provide the wrench, $\boldsymbol{F}_{\boldsymbol{b}}$ and $\boldsymbol{\tau}$, such that the control task is stable. This task is represented formally with a positive definite storage function as $W_{a}\left(\boldsymbol{g}_{\boldsymbol{b}}, \boldsymbol{V}_{\boldsymbol{b}}, \boldsymbol{q}, \dot{\boldsymbol{q}}\right)>0$. Then, the controller must ensure that

$$
\dot{W}_{a} \leq-\delta\left(\boldsymbol{g}_{\boldsymbol{b}}, \boldsymbol{V}_{\boldsymbol{b}}, \boldsymbol{q}, \dot{\boldsymbol{q}}\right), \quad \delta \geq 0
$$

Note that $W_{a}$ is only a general requirement, which holds for typical control designs and (24) ensures that the states of the servicer are bounded.

\footnotetext{
${ }^{2}$ For simplicity we consider that the force transmitted to the client includes already the transformation at its Com.
} 


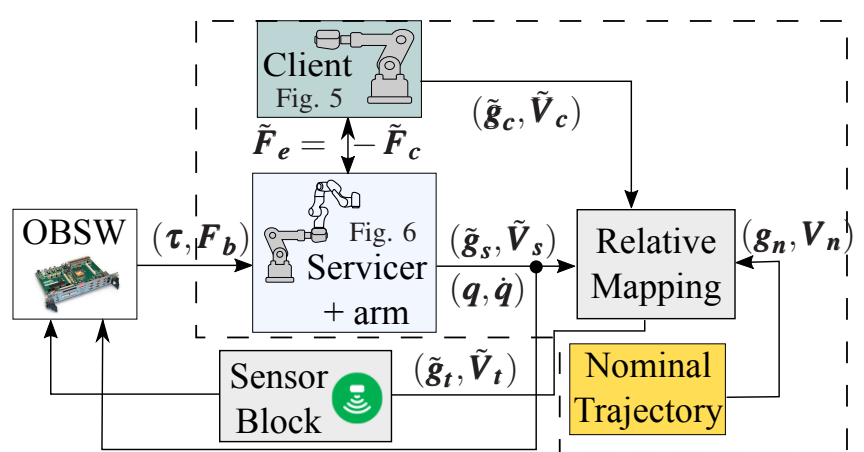

Fig. 7: Proposed architecture for simulating space robotic mission on-ground while exploiting the relative dynamics formulation.

\section{Stability analysis of the HiL simulation}

In this section, we prove stability of the HIL facility for two pertinent phases of on-orbit robotic missions, namely the approach and the grasping phase. For this, we exploit the stability analysis commonly used in the tracking control problem [19].

We show that the HIL facility is rendered to be stable relative to the nominal trajectory while exploiting the following advantages resulting from the proposed method. The Lagrangian (inertia) matching proposed in Sec. III-A - III-B, implies that the kinetic energies of the industrial robot on HIL are functions of the in-orbit inertias. Furthermore, the pose error definition in the OBSW controller directly depends on the states of the HIL robots because the proposed method preserves the geometric structures of the in-orbit elements without applying kinematic scaling. Hence, the storage function of the HIL facility is similar to $W_{a}$, however, using the actual HIL facility robot states, i.e. $\tilde{W}_{a} \equiv \tilde{W}_{a}\left(\boldsymbol{g}_{\boldsymbol{n}} \tilde{\boldsymbol{g}}_{\boldsymbol{s}}, \tilde{\boldsymbol{V}}_{\boldsymbol{s}}+\Delta \boldsymbol{V}_{\boldsymbol{s}}, \boldsymbol{q}, \dot{\boldsymbol{q}}\right)$.

\section{A. Approach Phase}

During the approach phase, no contact forces act on the system, i.e. $\boldsymbol{F}_{\boldsymbol{e}}$ and $\boldsymbol{F}_{\boldsymbol{t}}$ on the servicer and client, respectively, are zero. As a consequence of OBSW stability in (24), we get $\dot{\tilde{W}}_{a} \leq-\boldsymbol{\delta}\left(\boldsymbol{g}_{\boldsymbol{n}} \tilde{\boldsymbol{g}}_{\boldsymbol{s}}, \tilde{\boldsymbol{V}}_{\boldsymbol{s}}+\Delta \boldsymbol{V}_{\boldsymbol{s}}, \boldsymbol{q}, \dot{\boldsymbol{q}}\right)$. Hence, the HIL servicer states $\left(\boldsymbol{g}_{\boldsymbol{s}}, \boldsymbol{V}_{\boldsymbol{s}}\right)$ are stabilized (i.e., the equilibrium is stable) around $\left(\boldsymbol{g}_{n}^{-1},-\Delta \boldsymbol{V}_{\boldsymbol{s}}\right)$ and $(\boldsymbol{q}, \dot{\boldsymbol{q}})$ are stabilized around the origin. For the client HIL robot, the storage function is the kinetic energylike function, which is written as,

$$
\tilde{W}_{c}=\frac{1}{2}\left(\tilde{\boldsymbol{V}}_{\boldsymbol{c}}-\left(-\Delta \boldsymbol{V}_{\boldsymbol{c}}\right)\right)^{T} \boldsymbol{M}_{\boldsymbol{t}}\left(\tilde{\boldsymbol{V}}_{\boldsymbol{c}}-\left(-\Delta \boldsymbol{V}_{\boldsymbol{c}}\right)\right) .
$$

Since $\left(\tilde{\boldsymbol{V}}_{c}+\Delta \boldsymbol{V}_{\boldsymbol{c}}\right)=\tilde{\boldsymbol{V}}_{\boldsymbol{t}}$, using the passivity property, $\tilde{\boldsymbol{V}}_{\boldsymbol{t}}^{T}\left(\dot{\boldsymbol{M}}_{\boldsymbol{t}}-2 \boldsymbol{C}_{\boldsymbol{t}}\left(\tilde{\boldsymbol{V}}_{\boldsymbol{t}}\right)\right) \tilde{\boldsymbol{V}}_{\boldsymbol{t}}=0$, the resulting $\dot{W}_{c}=0$ is a loss-less system relative to the trajectory $-\Delta \boldsymbol{V}_{c}$.

\section{B. Grasping Phase}

In this phase, Assumption 3 holds i.e. $\boldsymbol{F}_{\boldsymbol{e}}=-\boldsymbol{F}_{\boldsymbol{t}}$ and the compound system (in Fig. 7 dashed-box) is represented by the storage function as $\tilde{W}_{t}=\tilde{W}_{a}+\tilde{W}_{c}$. Taking the time derivative of $\tilde{W}_{t}$ using (24), we obtain,

$$
\dot{\tilde{W}}_{t} \leq-\delta\left(\tilde{\boldsymbol{g}}_{\boldsymbol{b}}, \tilde{\boldsymbol{V}}_{\boldsymbol{b}}, \boldsymbol{q}, \dot{\boldsymbol{q}}\right)+\alpha\left(\tilde{\boldsymbol{V}}, \boldsymbol{q}, \dot{\boldsymbol{q}}, \tilde{\boldsymbol{F}}_{\boldsymbol{e}}, \tilde{\boldsymbol{V}}_{\boldsymbol{t}}\right),
$$

where $\alpha$ is a sign-indefinite function which captures the power injected due to the interaction wrench between the client's grappling point and the servicer's manipulator end-effector. Hence, for general contact, an injection of energy occurs in the last term of (26). In the particular case of an OBSW controller, which is passive with an external wrench input at the endeffector, (26) has a form,

$$
\dot{\tilde{W}}_{t} \leq-\boldsymbol{\delta}_{+}\left(\tilde{\boldsymbol{g}}_{\boldsymbol{b}}, \tilde{\boldsymbol{V}}_{\boldsymbol{b}}, \boldsymbol{q}, \dot{\boldsymbol{q}}\right)+\left[\begin{array}{lll}
\tilde{\boldsymbol{V}}_{\boldsymbol{b}}^{T} & \dot{\boldsymbol{q}} & \tilde{\boldsymbol{V}}_{\boldsymbol{t}}^{T}
\end{array}\right]\left[\begin{array}{c}
\boldsymbol{J}_{\boldsymbol{b}}^{T} \\
\boldsymbol{J}_{\boldsymbol{m}}^{T} \\
-\boldsymbol{I}
\end{array}\right] \tilde{\boldsymbol{F}}_{\boldsymbol{e}},
$$

where $\delta_{+}>0$. Given $\tilde{V}_{\boldsymbol{b}}$ and $\tilde{V}_{\boldsymbol{t}}$ as in (23) and considering that $\tilde{\boldsymbol{V}}_{\boldsymbol{a}}=\boldsymbol{J}_{\boldsymbol{b}} \tilde{\boldsymbol{V}}_{\boldsymbol{s}}+\boldsymbol{J}_{\boldsymbol{m}} \dot{\boldsymbol{q}}$ is the end-effector velocity on the HIL facility, since at the point of contact, $\boldsymbol{J}_{\boldsymbol{b}} \Delta \boldsymbol{V}_{\boldsymbol{s}}=\Delta \boldsymbol{V}_{\boldsymbol{c}}$, (27) is simplified as,

$$
\dot{\tilde{W}}_{t} \leq-\delta_{+}\left(\tilde{\boldsymbol{g}}_{\boldsymbol{b}}, \tilde{\boldsymbol{V}}_{\boldsymbol{b}}, \boldsymbol{q}, \dot{\boldsymbol{q}}\right)+\left(\tilde{\boldsymbol{V}}_{\boldsymbol{a}}-\tilde{\boldsymbol{V}}_{\boldsymbol{c}}\right)^{T} \tilde{\boldsymbol{F}}_{\boldsymbol{e}}
$$

Eq. (28) shows that a passivity map exists for the HIL facility robots as, $\tilde{\boldsymbol{F}}_{\boldsymbol{e}} \mapsto\left(\tilde{\boldsymbol{V}}_{\boldsymbol{a}}-\tilde{\boldsymbol{V}}_{\boldsymbol{c}}\right)$.

In the particular case of grasping, the gripper mounted on the manipulator arm is mechanically attached to the grappling point on the client, $\tilde{V}_{a}=\tilde{V}_{c}$, due to the gripper mechanical constraint. Therefore, (28) simplifies to $\dot{\tilde{W}}_{t} \leq-\boldsymbol{\delta}_{+}\left(\tilde{\boldsymbol{g}}_{\boldsymbol{b}}, \tilde{\boldsymbol{V}}_{\boldsymbol{b}}, \boldsymbol{q}, \dot{\boldsymbol{q}}\right)$. This implies that $\left(\tilde{\boldsymbol{g}}_{\boldsymbol{s}}, \tilde{\boldsymbol{V}}_{\boldsymbol{s}}\right)$ is stabilized around $\left(\boldsymbol{g}_{n}^{-1},-\Delta \boldsymbol{V}_{\boldsymbol{s}}\right)$ and $(\boldsymbol{q}, \dot{\boldsymbol{q}})$ is stabilized around the origin. Therefore, the end-effector velocity and the satellite velocity $\tilde{V}_{t}$ go to zero. This implies that the HIL client velocity $\tilde{V}_{c}$ is stabilized around the trajectory $-\Delta \boldsymbol{V}_{c}$. A corollary of the aforementioned observation is that the velocity of the servicer $\tilde{V}_{s}$ is stabilized around the client velocity $\tilde{V}_{c}$, i.e. the servicer and client have zero relative velocity on HIL.

\section{Boundedness of the industrial robots velocities}

To conclude stability (boundedness) of the velocity of the industrial robots, the nominal motion has to be appropriately designed. Specifically, if the nominal trajectory $\left(\boldsymbol{g}_{\boldsymbol{n}}, \boldsymbol{V}_{\boldsymbol{n}}\right)$ is chosen to be bounded as in (4), then the HIL robot velocities are also bounded, i.e.

$$
\begin{aligned}
& \left\|\tilde{\boldsymbol{V}}_{\boldsymbol{s}}\right\|=\left\|\tilde{\boldsymbol{V}}_{\boldsymbol{b}}-\Delta \boldsymbol{V}_{\boldsymbol{s}}\right\| \leq\left\|\tilde{\boldsymbol{V}}_{\boldsymbol{b}}\right\|+\left\|\Delta \boldsymbol{V}_{\boldsymbol{s}}\right\| \\
& \left\|\tilde{\boldsymbol{V}}_{\boldsymbol{c}}\right\|=\left\|\tilde{\boldsymbol{V}}_{\boldsymbol{t}}-\Delta \boldsymbol{V}_{\boldsymbol{c}}\right\| \leq\left\|\tilde{\boldsymbol{V}}_{\boldsymbol{t}}\right\|+\left\|\Delta \boldsymbol{V}_{\boldsymbol{c}}\right\|
\end{aligned}
$$

This is due to the boundedness of $\Delta \boldsymbol{V}_{\boldsymbol{s}}=\mathbf{A d}_{\boldsymbol{g}_{s}}^{-1} \boldsymbol{V}_{n}$ and $\Delta \boldsymbol{V}_{\boldsymbol{c}}=\mathbf{A d}_{\boldsymbol{g}_{\boldsymbol{c}}}^{-1} \boldsymbol{V}_{\boldsymbol{n}}$ during approach and post-grasp because $\tilde{\boldsymbol{g}_{\boldsymbol{s}}}=\boldsymbol{g}_{\boldsymbol{n}}^{-1} \tilde{\boldsymbol{g}_{\boldsymbol{b}}}$ and $\tilde{\boldsymbol{g}_{\boldsymbol{c}}}=\boldsymbol{g}_{n}^{-1} \tilde{\boldsymbol{g}_{\boldsymbol{t}}}$ are also bounded.

To summarize the above analysis, the servicer dynamics was stabilized using the OBSW controller with requirements as in (24), while the client dynamics remains lossless in Sec. IV-A. In the grasping phase, the states of both, the servicer and client are proved to be stable, as shown in Sec. IV-B. Note that $\boldsymbol{V}_{\boldsymbol{t}}$ remains bounded in both these cases. Therefore, an OBSW controller could also be designed in a way that the servicer tracks the moving satellite, see e.g. [20, Th. 2], [3]. Since the interaction wrench, $\boldsymbol{F}_{\boldsymbol{e}}$, is internal to the overall system, the stability properties presented in this section, will still hold relative to the client motion. As a consequence, for a bounded nominal motion, (29) holds and hence the HIL facility robots will have bounded velocities. 


\section{EXPERIMENTAL VALIDATION}

The proposed method was tested on the OOS-Sim HIL facility and the experimental results were verified by comparing with the results from the dynamics engine of the GNCDE software [16], which provides performance models of sensors, dynamics and actuators. The client satellite was initialized with an angular velocity of $-1 \mathrm{deg} / \mathrm{s}$ and the inertia parameters of the Envisat satellite were set. The unforced motion from (4) was chosen as the nominal trajectory. To verify the evolution of the unforced motion between the servicer and the client, the actuation commands of the OBSW controller in Fig. 7, i.e. $\left(\boldsymbol{F}_{\boldsymbol{b}}, \boldsymbol{\tau}\right)$ were set to zero. In Fig. 8 and Fig. 9, we compare the position and orientation, respectively, of the servicer Com with respect to the client Com, as were obtained from both, the GNCDE software (dashed) and the measurements derived from the industrial robots (blue). The orientation is computed from the relative rotation matrix and represented by the Euler angles (yaw, pitch and roll). In Fig. 9 only the yaw angle, $\psi$, is shown since the other two did not differ significantly. The maximum error was found to be within $1 \mathrm{~mm}$ and $0.002 \mathrm{rad}$, which proves the correctness of the proposed method. The low oscillation that occurs in Fig. 8 (see y-axis) is within the accuracy specification of the robot, and the frequency variation is due to a dependency on the velocity, which was increasing with time for this experiment.

For completeness, we show the validation results of an onboard sensor (i.e. the star tracker), which is usually exploited by the OBSW controller of the servicer. The comparison between the performance model running on the HIL and the GNCDE software is shown in Fig. 10. This sensor model exploits the states formulated in (23).
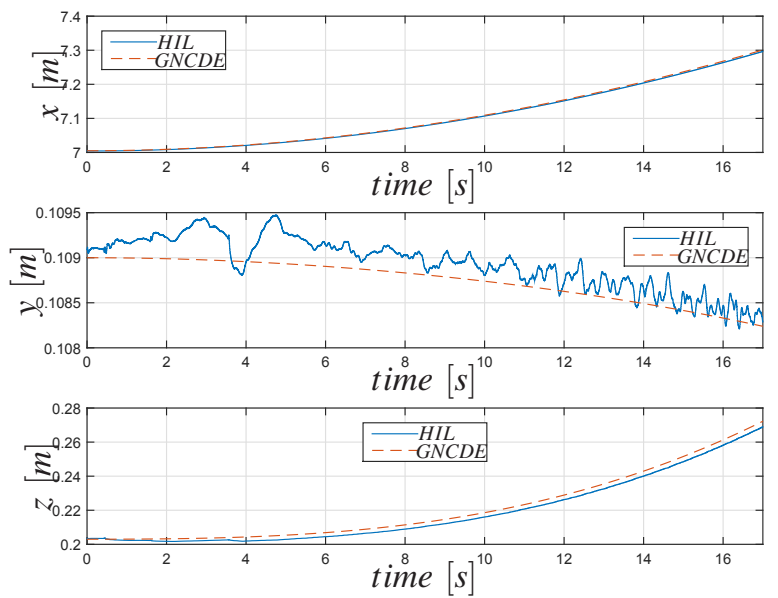

Fig. 8: Relative position between servicer and client in the OOS-Sim facility and comparison with GNCDE.

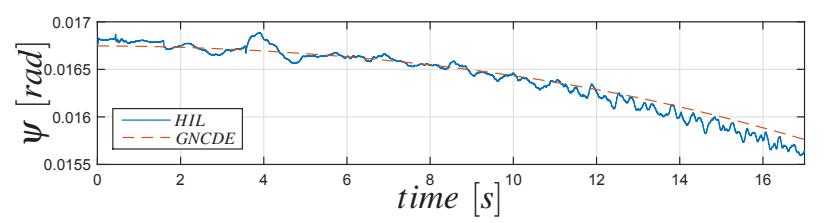

Fig. 9: Relative orientation between servicer and client in the OOSSim facility and comparison with GNCDE.
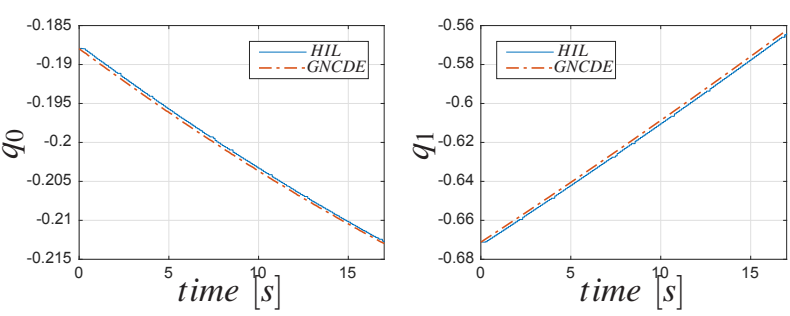

Fig. 10: Star Tracker outputs in the OOS-Sim facility and comparison with GNCDE for two quaternion components, $q_{0}, q_{1}$.

Hence, the validation of sensor performance concludes that the absolute servicer states are correctly mapped within the proposed relative dynamics formulation.

\section{Application: on-ground validation for the capture of Envisat}

The proposed method was applied for the on-ground validation of the capture of Envisat satellite within the ESA COMRADE project [3]. The facility was equipped with hardware elements representative of this mission study. The manipulator arm on the servicer was equipped with a gripper, which was used to grasp the Launch-adapter-ring profile attached to the client robot. The phases of the experiments are reported in Fig. 11.

The OBSW controller for the HIL validation was designed as a nonlinear compliant controller (see [3], §6.2.1), which provides the actuation, $\boldsymbol{\tau}$ and $\boldsymbol{F}_{\boldsymbol{b}}$ in Fig. 7 to perform the synchronization, approach and grasping phase of the client, i.e. Envisat satellite. Fig. 12 summarizes the experimental results. On the top line, the relative error of the manipulator and base is shown and on the bottom, the corresponding actuation torques for the manipulator arm and thrusters for the servicer base are shown. The client is initialized with an angular velocity of $-2.5 \mathrm{deg} / \mathrm{s}$ about its major axis of inertia and between $t=0-9 s$ the synchronization phase occurs, i.e., the servicer spacecraft regulates its pose with respect to the client. At $t=9.1 \mathrm{~s}$, the manipulator arm starts the approach and the grasp of the Envisat occurs at $32.6 s$ (see the dashed-line) in Fig. 12. The relative velocity of the servicer with respect to the client is shown in Fig. 13. It can be seen that before the grasping, the angular error is approximately $\pm 0.2 \mathrm{deg} / \mathrm{s}$ component-wise (see between $t=15-32.6 \mathrm{~s}$ ).

The validation of the controller for the capture of Envisat was feasible thanks to the proposed relative dynamics formulation. Commonly, a rigidization phase (see [3], §6.2.2) follows the grasping and the manipulator arm damps the remaining relative velocity, which is not considered within this experiment.
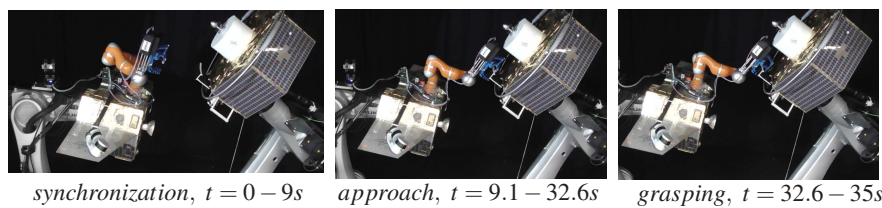

Fig. 11: Experiment for the robotic capture of Envisat (robot on the right) on the OOS-Sim facility. This experiment can be seen in the the video accompanying the paper. 

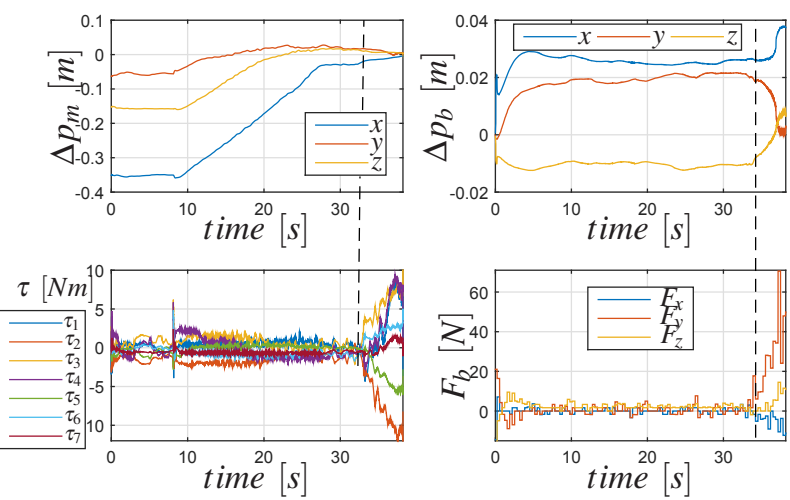

Fig. 12: Top: manipulator error $\Delta p_{m}$, and base error $\Delta p_{b}$. Bottom: servicer arm torques, $\tau$, and thruster force at the base, $\boldsymbol{F}_{\boldsymbol{b}}$. The dashed-line indicates the time on which the grasping occurs.

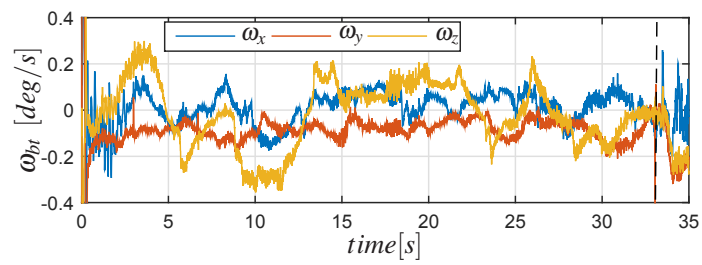

Fig. 13: Servicer angular velocity with respect to the client.

\section{Discussion AND CONCLUSION}

The proposed method exploits a relative dynamics formulation, which allows the on-ground simulation of a space robotic mission considering large satellite structures and velocities.

Stability was proved with respect to a nominal motion, which results in bounded velocities of the on-ground robotic facility while considering Assumption 1. However, if the timedelay in the control loop of a generic admittance-controlled robot is not negligible, the loss of passivity due to this timedelay can be compensated with a passivity controller, as shown e.g. in [18], [21]. A technical requirement of the proposed approach is the increase in computations for the HIL simulation in real-time. This factor is due to the numerical integration of two dynamics models for each spacecraft, namely (2) and (11) for the client and (13) and (18) for the servicer.

We assumed that the manipulator arm on ground has the same kinematics and inertial parameters of the manipulator arm in orbit (Assumption 2). Although this assumption simplifies the analysis, it is not restrictive from an engineering point of view. Several physical aspects might not be identical when different manipulators are considered, e.g. joints friction, motors frequency and accuracy or torque limits. These factors might affect the response of the manipulator controller and, consequentially, the validation of the space robotic mission. Assumption 3 simply states that the external contact is considered only between the servicer manipulator end-effector and the grasping point of the client satellite.

The proposed method was pivotal to the evaluation of a control algorithm for the capture of the tumbling Envisat satellite. The method was validated experimentally on ground within a space mission study.

\section{ACKNOWLEDGMENT}

The authors thank P. Colmenarejo, N. Santos, J. Branco (GMV), J. Jaworski (PIAP) and G. Visentin (ESA) for projectrelated support.

\section{REFERENCES}

[1] S. Jaekel et al., "Design and operational elements of the robotic subsystem for the e.deorbit debris removal mission," Frontiers in Robotics and AI, vol. 5, p. 100, 2018.

[2] J. Telaar et al., "Gnc architecture for the e.deorbit mission," 7th European Conference for Aeronautics and Space Science, 2017.

[3] P. Colmenarejo et al., "Methods and outcomes of the comrade project design of robust combined control for robotic spacecraft and manipulator in servicing missions: Comparison between hinf and nonlinear lyapunovbased approaches," in 69th International Astronautical Congress, 2018.

[4] A. Flores-Abad, O. Ma, K. Pham, and S. Ulrich, "A review of space robotics technologies for on-orbit servicing," Progress in Aerospace Sciences, vol. 68, pp. 1 - 26, 2014.

[5] J. Artigas et al., "The OOS-SIM: An on-ground simulation facility for on-orbit servicing robotic operations," in Robotics and Automation (ICRA), IEEE International Conference on, May 2015, pp. 2854-2860.

[6] F. Aghili and M. Namvar, "Scaling inertia properties of a manipulator payload for 0-g emulation of spacecraft." I. J. Robotic Res., vol. 28, no. 7, pp. 883-894, 2009.

[7] F. Aghili and J. Piedboeuf, "Hardware-in-loop simulation of robots interacting with environment via algebraic differential equation," in IEEE/RSJ International Conference on Intelligent Robots and Systems (IROS), vol. 3, Oct 2000, pp. 1590-1596 vol.3.

[8] R. Lampariello et al., "Tracking control for the grasping of a tumbling satellite with a free-floating robot," IEEE Robotics and Automation Letters, vol. 3, no. 4, pp. 3638-3645, Oct 2018.

[9] T. Boge et al., "EPOS - Using Robotics for RvD Simulation of OnOrbit Servicing Missions," AIAA Modeling and Simulation Technologies Conference, aug 2010.

[10] H. Shimoji et al., "Simulation system for a space robot using 6 axis servos," IFAC Proceedings Volumes: IFAC Symposium on Automatic Control in Aerospace, vol. 22, no. 7, pp. 115 - 120, Jul 1989.

[11] R. Takahashi et al., "Hybrid simulation of a dual-arm space robot colliding with a floating object," IEEE International Conference on Robotics and Automation (ICRA), pp. 1201-1206, 2008.

[12] D. E. Chang and J. E. Marsden, "Reduction of controlled lagrangian and hamiltonian systems with symmetry," SIAM Journal of Control and Optimization, vol. 43, no. 1, pp. 277-300, Jan 2004.

[13] N. Hogan, "Impedance control: An approach to manipulation: Part II - Implementation," Journal of Dynamic System, Measurement and Control, vol. 107(1):8-16, 1985.

[14] C. Carignan, N. Scott, and S. Roderick, "Hardware-in-the-loop simulation of satellite capture on a ground-based robotic testbed," International Symposium on Artificial Intelligence, Robotics and Automation in Space (i-SAIRAS), 2014.

[15] S. K. Agrawal et al., "A new laboratory simulator for study of motion of free-floating robots relative to space targets," IEEE Transactions on Robotics and Automation, vol. 12, no. 4, pp. 627-633, Aug 1996.

[16] P. Colmenarejo et al., "Gncde: An integrated gnc development environment," 6th International ESA Conference on Guidance, Navigation and Control Systems, 2006.

[17] H. Mishra et al., "A nonlinear observer for free-floating target motion using only pose measurements," in American Control Conference (ACC), 2019, pp. 1114-1121.

[18] M. De Stefano, R. Balachandran, and C. Secchi, "A passivity-based approach for simulating satellite dynamics with robots: Discrete-time integration and time-delay compensation," IEEE Transactions on Robotics, vol. 36, no. 1, pp. 189-203, 2020.

[19] M. De Stefano et al., "Multi-rate tracking control for a space robot on a controlled satellite: A passivity-based strategy," IEEE Robotics and Automation Letters, vol. 4, no. 2, pp. 1319-1326, April 2019.

[20] H. Mishra et al., "A geometric controller for fully-actuated robotic capture of a tumbling target," in 2020 American Control Conference (ACC), 2020, pp. 2150-2157.

[21] M. De Stefano et al., "Reproducing physical dynamics with hardwarein-the-loop simulators: A passive and explicit discrete integrator," in Robotics and Automation (ICRA), 2017 IEEE International Conference on, May 2017, pp. 5899-5906. 